\title{
Research on Case-based Teaching of Engineering Software Based on Mechanical Applied Talents Training
}

\author{
Shukun Gan
}

\author{
College of Mechanical and Electrical Engineering, Jilin Institute of Chemical Technology, Jilin \\ 132022, China \\ 49141544@qq.com
}

Keywords: Applied talent training; Engineering software; LPG tank design; Instantiation teaching

\begin{abstract}
In order to strengthen the comprehensive application ability of engineering software for mechanical application talents.By summarizing the teaching experience and using the example teaching, the UG software 3D modeling of the LPG storage tank and the design and analysis of the working platform of ANSYS Workbench are taken as an example.The tank, manhole, saddle support, etc. were designed, analyzed and discussed.The results show that the engineering design method of engineering software is reliable and effective, and can be used as the scientific basis for engineering practice. The instant teaching of engineering software can effectively improve the overall quality and ability of students' engineering design, realize the training goal of engineering quality education of applied innovative talents, and has important theoretical guiding significance and application promotion value.

In recent years, with the rapid development of mechanical science, the mechanical majors of higher education institutions in China have implemented a wide-calibre talent training model, and the application of mechanical professional talents. With the advent of computer-aided design (CAD) and computer-aided engineering (CAE) technologies, the application of CAD/CAE/CAM and other engineering software to solve engineering problems has become an important part of modern design methods.

This paper combines UG, ANSYS Workbench and other engineering software to apply the instantiation teaching of liquefied petroleum gas (LPG) storage tank design, so as to achieve the training goal of application talents and engineering quality education.
\end{abstract}

\section{Process Design of Liquefied Petroleum Gas Storage Tank}

Liquefied petroleum gas (LPG) storage tanks are widely used in various industrial and civil fields as a special pressure vessel.However, due to flammable and explosive, the safety of tank design and manufacture must be fully guaranteed to avoid unnecessary casualties and major property losses [1].Through the optimization design and analysis of modern advanced CAD/CAE software, it is possible to improve safety and achieve energy saving and consumption reduction as much as possible [2, 3, 4].In the design process, the horizontal LPG storage tanks are mainly studied for structural and safety considerations. The ANSYS software was used to design and analyze the relevant structure of the tank body, manhole joint reinforcement and saddle support, and the corresponding analytical results with certain value were obtained [5].

According to the relevant design condition parameters given in Table 1, and the provisions of the "Safety Technical Supervision Regulations for Fixed Pressure Vessels". Firstly, the design is carried out by the conventional design method. The original design condition medium is liquefied petroleum gas, and the saturated vapor pressure at $50{ }^{\circ} \mathrm{C}$ is greater than $1.62 \mathrm{MPa}$, and the design pressure should be $1.05 \sim 1.1$ times the maximum working pressure due to the safety valve on the storage tank.[1] the final design pressure was set at 2.16 MPa.The specific conditions are working pressure $1.96 \mathrm{MPa}$, design temperature $50^{\circ} \mathrm{C}$, nominal diameter $2000 \mathrm{~mm}$, volume $20 \mathrm{~m}^{3}$, loading coefficient 0.9 .The liquefied petroleum gas storage tank belongs to the class III pressure vessel and is flammable and explosive. The main material of the equipment is selected as Q345R (hot rolled steel sheet) and $16 \mathrm{MnII}$ forging. 
Table 1 Original design data

\begin{tabular}{cll}
\hline No. & \multicolumn{1}{c}{ Item } & \multicolumn{1}{c}{ Parameter } \\
\hline 1 & Name & LPG Storage tank \\
2 & Use & LPG Reserve station \\
3 & Working pressure & $1.96 \mathrm{MPa}$ \\
4 & Set tempreture & $50^{\circ} \mathrm{C}$ \\
5 & Unit volume filling & $0.42 \mathrm{t} / \mathrm{m}^{3}$ \\
6 & Nominal diameter & $2000 \mathrm{~mm}$ \\
7 & Volume & $20 \mathrm{~m}^{3}$ \\
8 & Loading factor & 0.9 \\
9 & Working medium & LPG (Flammable) \\
10 & Other requirements & $100 \%$ Nondestructive testing \\
\hline
\end{tabular}

\section{UG NX Software 3D Modeling}

Based on conventional design results, a three-dimensional model of the tank was obtained by three-dimensional modeling design by UG NX software.In order to facilitate the subsequent analysis and processing of ANSYS software, a tank body is created as shown in Fig. 1(a), a manhole joint is shown in Fig. 1(b), (c), and a saddle support is shown in Fig. 1(d). And exported in IGES file format.
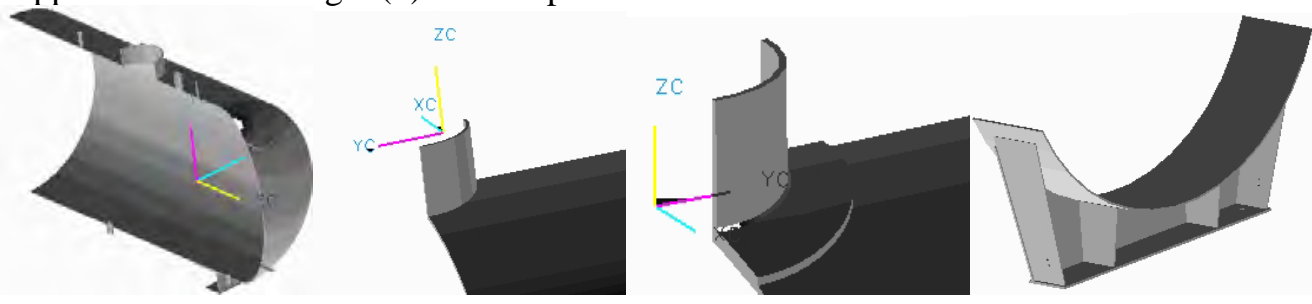

(a) 1/4 Tank model (F type)

(b) 1/4 Manhole takeover without reinforced

(c) 1/4 Manhole take-over (with reinforcing ring)

(d) Saddle

Figure 1 Model of the tank component for UG NX builds

\section{ANSYS Finite Element Analysis}

ANSYS software is one of the advanced CAE tools in modern product design , and is a large-scale general finite element analysis software. ANSYS Workbench is a module of ANSYS,

the ANSYS series products are integrated into the simulation platform to enable seamless transfer and sharing of data [6].

The discontinuous zone of the pressure vessel is often the high stress zone of the pressure vessel,in order to complete the process and facilitate the inspection and maintenance, the petrochemical equipment must be equipped with an interface, which makes the pressure vessel must be opened, and the opening breaks the continuity of the casing material, which will cause stress concentration near the edge of the opening [ 7,8].Therefore, the ANSYS software is used to analyze and discuss the tank, manhole reinforcement and saddle.

Tank Analysis. Since the open joint is mostly on the left side of the tank, and the diameter of the left joint is representative.Therefore, in order to reduce the computational analysis time, the following models are modeled $1 / 4$ on the left side of the tank. It is assumed here that the manhole nozzle is not reinforced.

Import the IGES file exported from the tank model into ANSYS Workbench.after setting the material properties (Young's modulus of elasticity, Poisson's ratio, density, etc.), select the static structure analysis, set the corresponding boundary conditions in the property window, including the load and constraints, and insert the result output to solve.

Pressure vessels fail because of an excessive or too small amount of stress, strain, or amount associated with them.Therefore, this design analysis selects stress, strain, and overall deformation.The analysis only selects the overall deformation of the tank. First, the grid division of the tank is shown in Fig. 2. After the software analysis, the analysis result of the overall 
deformation shown in Fig. 3 can be obtained.

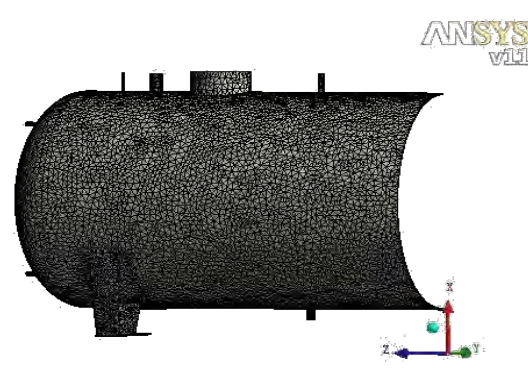

Figure 2 Grid diagram of tank

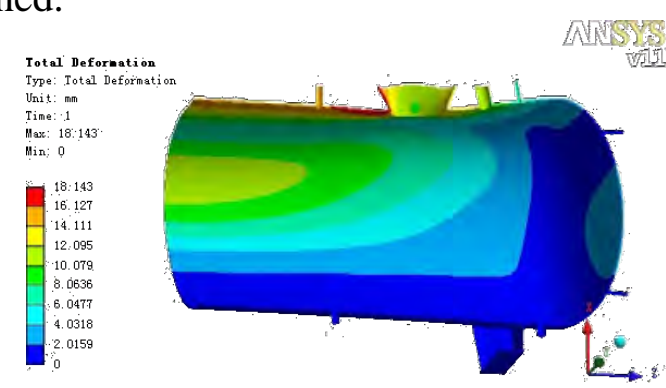

Figure 3 Overall deformation of the tank

The following conclusions are drawn from the results of the analysis, The body deformation has been flexed at the manhole joint (the largest deformation in this experimental test is $18.143 \mathrm{~mm}$ ), and the actual maximum deformation of the project may be around $18 \mathrm{~mm}$.In combination with the actual project, it should be noted that the correction is considered at this maximum deformation to determine whether reinforcement is required.

Manhole Takeover Analysis. Due to the opening of the manhole, the strength of the container wall is weakened.At the joint between the casing and the joint, the continuity of the structure is destroyed, and high local stress is generated, and the joint needs to be reinforced.When analyzing and designing, it is divided into three parts: the take-over, the reinforcing ring and the container shell. The balance and deformation coordination equation of the force is established at the joint to solve the displacement and stress at the joint.

The design has an inner diameter of $2000 \mathrm{~mm}$, a length of $6400 \mathrm{~mm}$, a thickness of $16 \mathrm{~mm}$, a manhole joint length of $190 \mathrm{~mm}, \varphi 530 \times 12$, a reinforcing ring outer diameter of $840 \mathrm{~mm}$, an inner diameter of $534 \mathrm{~mm}$, and a reinforcing thickness of $14 \mathrm{~mm}$, for example.In order to improve the efficiency of the subsequent analysis and calculation, the analysis of the manhole takeover with or without the reinforcement circle is carried out with the $1 / 4$ model of the takeover, and the model is shown in Fig. 2 b and c.

This section only analyzes the effects of the presence or absence of a reinforcing ring.In the case of reinforcing the ring, the reinforcing ring is connected by welding, and considering the influence of the welding, the corresponding boundary condition is given in the modeling. Here, only the stress output items are taken, and the stress cloud map analysis results shown in Fig. 4 and Fig. 5 are obtained in two cases.

The following conclusions can be drawn from the results of the analysis. The stress concentration between the take-over and the casing is real (the peak stress is $736.82 \mathrm{MPa}$ when there is no reinforcing ring in the test results of this experiment). Compared with the joint without the reinforcing ring structure, the reinforcing ring relieves the stress concentration, and the peak stress is reduced by about $60 \%$ to $281.59 \mathrm{MPa}$. In the yield strength range, the strength failure does not occur due to the stress concentration of the take-over. The stress is gradually decreasing along the edge of the joint between the joint and the shell, which fully reflects the edge effect.

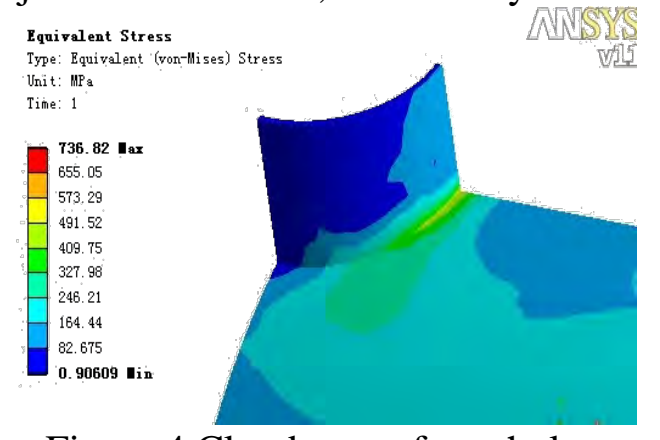

Figure 4 Cloud map of manhole nozzle without reinforcing stress 


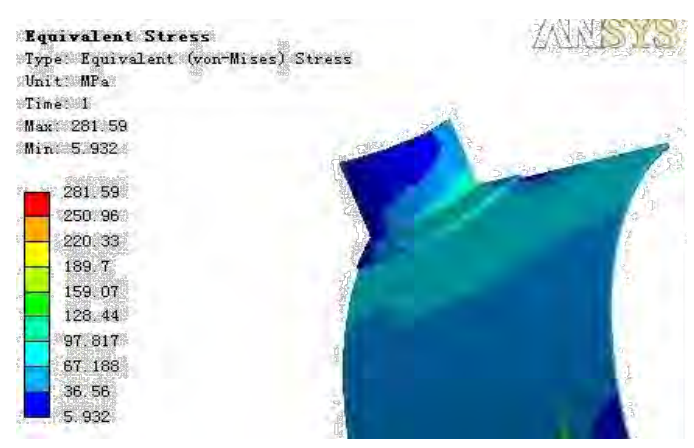

Figure 5 Cloud map of manhole nozzle with reinforced stress

Saddle Support Analysis. The pressure vessel has compressive stress in a local area. When the compressive stress exceeds the critical value, the structural instability will occur. The conventional design calculates that the load on the saddle is $285.796 \mathrm{KN}$, and the allowable load on the saddle is $\mathrm{Q}=300 \mathrm{KN}$. The fixed saddle is a three-dimensional finite element analysis for the study object. The model is shown in Figure 1(d).

Steel Q235R has a Young's modulus E=2.0 $\times 105 \mathrm{MPa}$, Poisson's ratio=0.3, yield limit $=235 \mathrm{MPa}$, select static structure analysis, set boundary conditions in the property window, consider the maximum equipment weight, horizontal force, radial reaction force, etc. when the tank hydraulic test is carried out $[7,8]$. Considering the design to select the stability of the saddle support itself, the output of the analysis results is von-Mises stress distribution as shown in Figure 6, the overall deformation is shown in Figure 7.

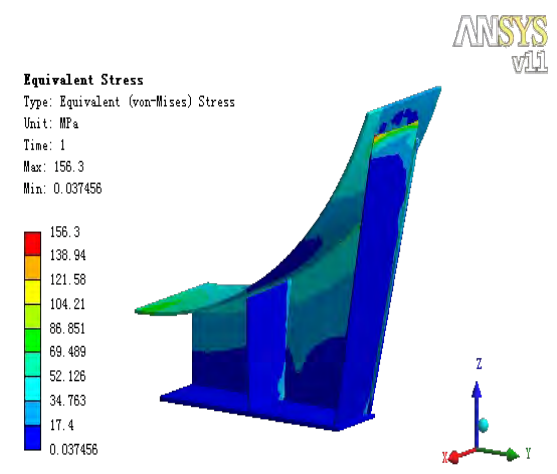

Figure 6 Stress distribution of saddle support von-Mises

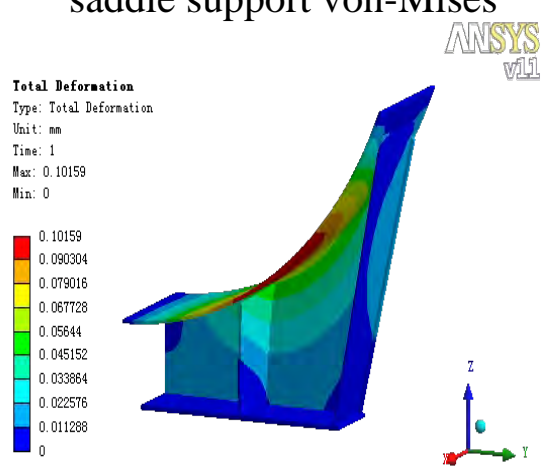

Figure 7 Overall deformation

of saddle support

The following conclusions can be drawn from the results of the analysis, the high stress area of the saddle is distributed on the backing plate, the maximum stress in the saddle stress distribution cloud diagram is $156.3 \mathrm{MPa}$, with an average of $70 \mathrm{MPa}$ or less, within the yield strength range; the maximum deformation of the saddle is $0.10159 \mathrm{~mm}$.

\section{Conclusion}

Through the optimized design and analysis of LPG storage tanks with CAD/CAE engineering software such as UG and ANSYS, through the optimized design and analysis of LPG storage tanks by CAD/CAE engineering 
software such as UG and ANSYS, valuable and effective data design results can be obtained, the design cycle and design cost can be shortened, and satisfactory design results can be obtained. achieve twice the result with half the effort. At the same time, the safety and rationality of the design are guaranteed, and an important scientific basis is obtained [9, 10]. Taking engineering examples as teaching research objects, using CAD/CAE and other engineering software to optimize design and analysis of mechanical products and equipment, it is closer to engineering practice, enhance students' perceptual and rational understanding, effectively improve teaching quality and improve students' comprehensive quality of engineering design. And the ability to achieve the training goal of engineering quality education for applied innovative talents.

\section{References}

[1] W.D.Sun,J.M.Fang: Industrial Safety and Environmental Protection,Vol.35(2009)No.12,p.44-47. (In Chinese)

[2] Q.W.Dong,M.S.Liu and H.L.Cao: Chemical Progress,(2003)No.01,p.71-75.(In Chinese)

[3] L.S.Wang: Mechanical Engineering and Automation,(2014)No.01,p.218-219. (In Chinese)

[4] TSGR0004-2009, Fixed Pressure Vessel Safety Technical Supervision Procedures. (In Chinese)

[5] Z.B.Zhu: Equipment Manufacturing Technology,(2004)No.01,p.16-20. (In Chinese)

[6] J.J.Wang: ANSYS11.0 Structure and Thermodynamic FiniteElement Analysis Example Tutorial(Mechanical Industry Press,China 2008),p.98. (In Chinese)

[7] Y.Dan,S.Y,Pei: Chemical Machinery,(2007)No.06,p.329-333. (In Chinese)

[8] X.Y.Zhang, China Chemical Equipment,(2005)No.03,p.19-21. (In Chinese)

[9] X.L.Wang: Journal of Jilin Institute of Chemical Technology,(2016)No.01,p.31-34. (In Chinese)

[10] F.R.Lu: Journal of Jilin Institute of Chemical Technology,(2015)No.08,p.46-49. 